\title{
Effect of point defects on the optical and transport properties of $\mathrm{MoS}_{\mathbf{2}}$ and $\mathrm{WS}_{2}$
}

\author{
Shengjun Yuan, ${ }^{1,{ }^{*}}$ Rafael Roldán, ${ }^{2, \dagger}$ M. I. Katsnelson, ${ }^{1}$ and Francisco Guinea ${ }^{2}$ \\ ${ }^{1}$ Radboud University of Nijmegen, Institute for Molecules and Materials, Heijendaalseweg 135, 6525 AJ Nijmegen, The Netherlands \\ ${ }^{2}$ Instituto de Ciencia de Materiales de Madrid, CSIC, Cantoblanco E28049 Madrid, Spain \\ (Received 14 April 2014; revised manuscript received 20 June 2014; published 2 July 2014)
}

\begin{abstract}
Imperfections in the crystal structure, such as point defects, can strongly modify the optical and transport properties of materials. Here, we study the effect of point defects on the optical and dc conductivities of single layers of semiconducting transition metal dichalcogenides with the form $M \mathrm{~S}_{2}$, where $M=$ Mo or W. The electronic structure is considered within a six band tight-binding model, which accounts for the relevant combination of $d$ orbitals of the metal $M$ and $p$ orbitals of the chalcogen $\mathrm{S}$. We use the Kubo formula for the calculation of the conductivity in samples with different distributions of disorder. We find that $M$ and/or S defects create midgap states that localize charge carriers around the defects and which modify the optical and transport properties of the material, in agreement with recent experiments. Furthermore, our results indicate a much higher mobility for $p$-doped $\mathrm{WS}_{2}$ in comparison to $\mathrm{MoS}_{2}$.
\end{abstract}

DOI: 10.1103/PhysRevB.90.041402

PACS number(s): 73.63.-b, 71.23.-k, 78.20.-e, 78.55.-m

Introduction. The mobility of current single-layer crystals of transition metal dichalcogenides (TMD) is highly dependent on the screening environment and is limited by the presence of defects in the samples. The existence of defects in the chemical and structural composition of those materials can influence their optical and transport properties, as revealed by recent experimental results. A broad peak at $\sim 700 \mathrm{~nm}$ $(\sim 1.77 \mathrm{eV})$ in the optical spectrum of bilayer $\mathrm{MoS}_{2}$ has been associated to impurities [1], whereas the mobility of multilayer samples has been shown to highly depend on the substrate and dielectric effects [2]. Vacancy defects in the crystal, which can be created by means of thermal annealing and $\alpha$-particle [3] or electron beam irradiation [4,5], trap free charge carriers and localize excitons, leading to new peaks in the photoluminescence spectra [3]. Recent experiments [5] show that the density of sulfur vacancies in $\mathrm{MoS}_{2}$ is of the order of $10^{13} \mathrm{~cm}^{-2}$, corresponding to an average defect distance of about $1.7 \mathrm{~nm}$. The existence of line defects, which separate patches or islands where the layer direction is opposite to its surrounding, can lead to changes in the carrier mobility [6], and the importance of short-range disorder has been proposed as the main limitation for the mobility of chemical vapor deposition grown single-layer $\mathrm{MoS}_{2}[7,8]$.

Therefore, it is necessary to understand the effect of impurities in the optical and transport properties of TMD, as a first step to exploit the controlled creation of defects as a route to manipulate their electronic properties. There are several theoretical works which have studied this problem using ab initio methods [6,9-15]. However, the simulation of realistic disordered samples of TMD with a random distribution of defects is extremely expensive computationally for density functional theory methods, since they require a very large unit cell in the calculation. In this Rapid Communication we follow an alternative route and perform a systematic study of the density of states, optical and dc conductivities of single layers of $\mathrm{MoS}_{2}$ and $\mathrm{WS}_{2}$ in the presence of point defects, by means of a real space tight-binding (TB) model for large systems,

\footnotetext{
*s.yuan@science.ru.nl

†rroldan@icmm.csic.es
}

containing millions of atoms. In our simulations, Mo/W and $\mathrm{S}$ point defects are introduced by elimination of atoms which are randomly distributed over the sample. This method allows us to study point defects such as unreconstructed vacancies, chemically bonded atoms or molecules, and strong substitutional defects which prevent the electronic hopping to the neighbors. We also consider clusters of point defects. We use a TB model that considers the relevant orbital contribution in the valence and conduction bands, as well as the effect of spin-orbit coupling (SOC) [16,17]. The optical and electronic properties are obtained numerically by using the tight-binding propagation methods (TBPM) [18-21]. Our results show that point defects create midgap states whose energy depends on the specific impurities. We show that optical transitions involving the impurity bands lead to a background contribution in the photoconductivity at low energies, in agreement with recent experiments [22]. We further calculate the dc conductivity of disordered $\mathrm{MoS}_{2}$ and $\mathrm{WS}_{2}$, finding that the impurity states do not contribute to the conductivity within the gap, whereas they lead to a depletion of it outside the gap.

Method. Transition metal dichalcogenides such as $\mathrm{MoS}_{2}$ and $\mathrm{WS}_{2}$ are composed, in its bulk configuration, of twodimensional S- $M$-S layers $(M=\mathrm{Mo}, \mathrm{W})$ stacked on top of each other, coupled by weak van der Waals forces. The transition metal atoms $M$ are ordered in a triangular lattice, each of them bonded to six S atoms located in the top and bottom layers, forming a sandwiched material. Similarly as in graphene, the weak interlayer coupling makes it possible to exfoliate this material down to a single layer [23]. The electronic band structure of $\mathrm{MoS}_{2}$ changes from an indirect band gap for multilayer samples, to a direct gap semiconductor for single layers $[16,24]$. We consider a six-band tight-binding model which contains the proper orbital combination that contributes to the valence and conduction bands of $M \mathrm{~S}_{2}$ : three $d$ orbitals of the transition metal $\left(d_{x y}, d_{x^{2}-y^{2}}\right.$, and $\left.d_{3 z^{2}-r^{2}}\right)$ as well as the symmetric (antisymmetric) combination of the $p_{x}, p_{y}\left(p_{z}\right)$ orbitals of the top and bottom chalcogen atoms $[16,25]$. The base vector can be written as

$$
\phi_{i}^{\dagger}=\left(d_{i, 3 z^{2}-r^{2}}^{\dagger}, d_{i, x^{2}-y^{2}}^{\dagger}, d_{i, x y}^{\dagger}, p_{i, x, A}^{\dagger}, p_{i, y, A}^{\dagger}, p_{i, z, S}^{\dagger}\right),
$$



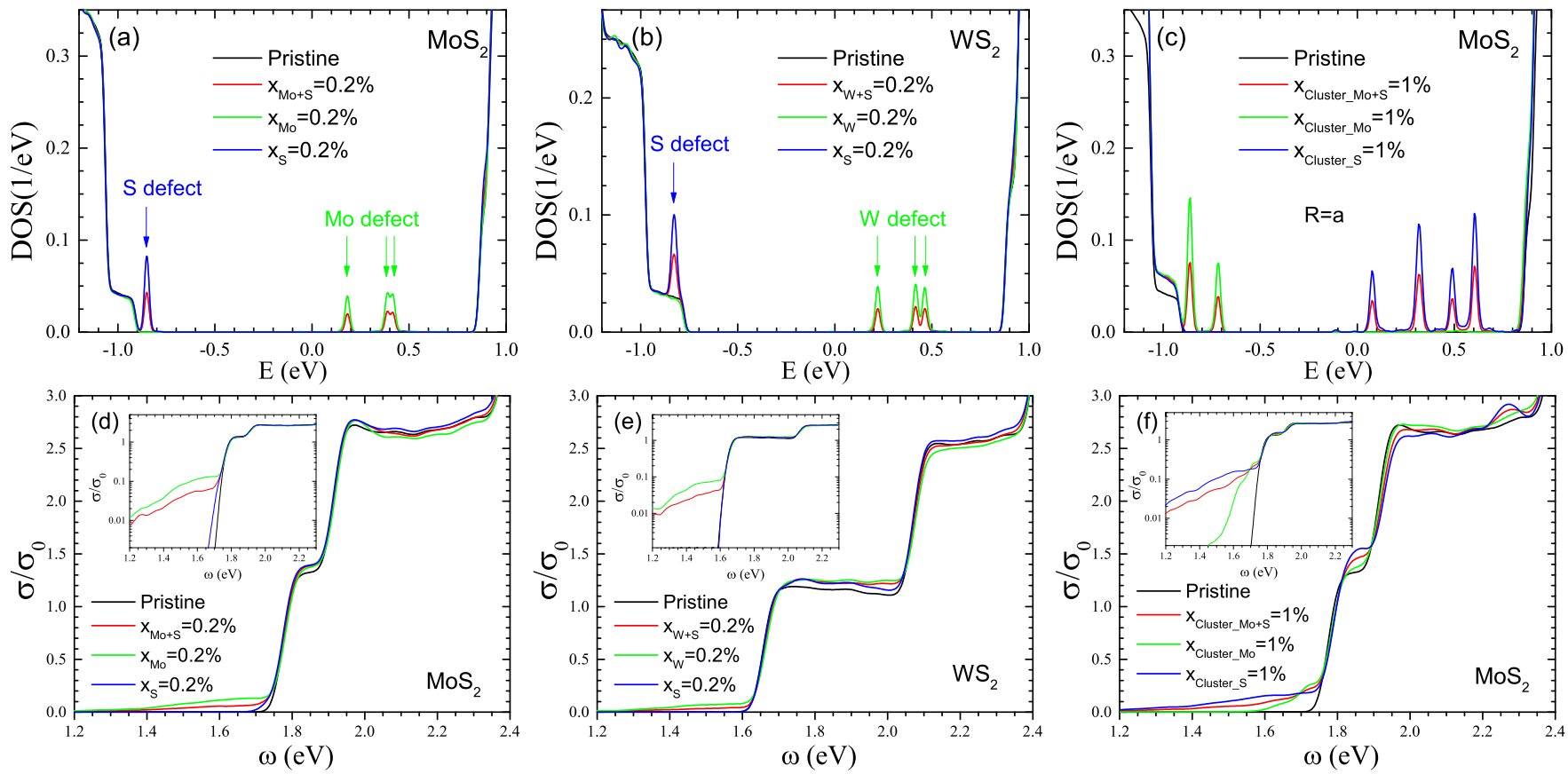

FIG. 1. (Color online) DOS of $\mathrm{MoS}_{2}$ (a) and $\mathrm{WS}_{2}$ (b) for different types of single point defects, and for MoS 2 (c) with clusters of defects with $R=a$ (the labels in the subscript of the concentration $x$ indicate the center of the cluster). The peaks in the DOS associated to midgap bands due to $\mathrm{S}$ or Mo/W defects are marked by arrows. Optical conductivity (in units of $\sigma_{0}=\pi e^{2} / 2 h$ ) for $\mathrm{MoS}_{2}$ (d) and WS $(\mathrm{e})$ with single defects, and for $\mathrm{MoS}_{2}$ with clusters of defects, for the same concentration of defects as in (a)-(c). The insets show the same plots in a logarithmic scale.

where $\quad p_{i, o, S}^{\dagger}=\left(p_{i, o, t}^{\dagger}+p_{i, o, b}^{\dagger}\right) / \sqrt{2}, \quad p_{i, o, A}^{\dagger}=\left(p_{i, o, t}^{\dagger}-\right.$ $\left.p_{i, o, b}^{\dagger}\right) / \sqrt{2}, o=x, y, z$ and the subscripts $t$ and $b$ refer to the top and bottom $\mathrm{S}$ layers, respectively.

We also consider the intra-atomic spin-orbit coupling $\sum_{a} \lambda_{a} \hat{\mathbf{L}}_{a} \cdot \hat{\mathbf{S}}_{a}$, where $a=M, \mathrm{~S}$ accounts for both the transition metal $M$ as well as the chalcogen atom $\mathrm{S}, \lambda_{a}$ is the corresponding intra-atomic SO interaction, $\hat{\mathbf{L}}$ is the atomic angular momentum operator, and $\hat{\mathbf{S}}=\hbar \hat{\boldsymbol{\sigma}}$ is the spin operator. The optical and electronic properties, such as density of states (DOS), quasieigenstates, and optical and dc conductivities, are obtained numerically by using the TBPM [18-21] (more details can be found in the Supplemental Material [26]).

Results and discussion. The effect of point defects in the DOS of $\mathrm{MoS}_{2}$ and $\mathrm{WS}_{2}$ are shown in Figs. 1(a)-1(c). The defect concentration $(0.2 \%)$ for single point defects in Figs. 1(a) and 1(b) is chosen to be of the same order as the intrinsic vacancies observed in recent experiments [5]. For clean samples (black lines), the DOS has a gap $\Delta$ which corresponds to the well known direct gap of single-layer samples at the $K$ points of the Brillouin zone (BZ). Defects in the samples lead to the appearance of a series of peaks in the gapped region of the DOS, which are associated to the creation of midgap states localized around the defects, whose energy and strength depends on the specific missing atoms, their concentration, as well as the specific arrangement of the point defects as individual missing atoms [Figs. 1(a) and $1(\mathrm{~b})]$ or in clusters of point defects with variable radius [Fig. 1(c)]. For the same concentration of defects, isolated point defects modify the DOS more strongly than clusters of defects. This is the reason why we show results for $0.2 \%$ of single defects, and $1 \%$ of cluster of defects with a radius
$R=a$ [26]. The impurity states also have an important effect on the optical conductivity [Figs. 1(d) and 1(e)]. First, let us consider the case of undoped and clean $\mathrm{MoS}_{2}$ and $\mathrm{WS}_{2}$. Since single layers of those TMD are direct gap semiconductors, the only optical transitions allowed at low energies are two sets of interband transitions with $\omega \geqslant \Delta$ from the edges of the SOC split valence bands to the conduction band at the $K$ and $K^{\prime}$ points of the BZ [27,28]. Those transitions lead to the $A$ and $B$ absorption peaks observed in photoluminescence experiments [22], and the SOC splitting of the valence band manifests itself in the optical conductivity through the steplike feature of $\sigma(\omega)$ that can be seen by the black lines (for pristine $\mathrm{MoS}_{2}$ and $\mathrm{WS}_{2}$ ) of Figs. 1(d)-1(f). This feature is especially visible for $\mathrm{WS}_{2}$ due to the strong SOC associated to the heavy $\mathrm{W}$ atom [17], which leads to a plateaulike feature for $\sigma(\omega)$ of $\sim 400 \mathrm{meV}$, in agreement with the energy separation between the spin polarized valence bands. The existence of defects in the sample leads to flat midgap bands which activate new optical transitions with $\omega<\Delta$ in the optical spectrum. Most importantly, these new optical transitions lead to a background contribution which appears in the optical conductivity at low energies, as it can be seen in Figs. 1(d)-1(f) for different concentrations of defects, suggesting that resonant impurities, like the defects studied in this work, could have a relevant contribution to the optical spectrum of TMDs [22]. Furthermore, it is interesting to note that this background contribution due to disorder resembles that observed in the optical conductivity of highly doped graphene [29].

Our results for the dc conductivities are shown in Fig. 2, which demonstrate a significant asymmetry between electrons and holes, in reasonable agreement with experiments [2] (note 

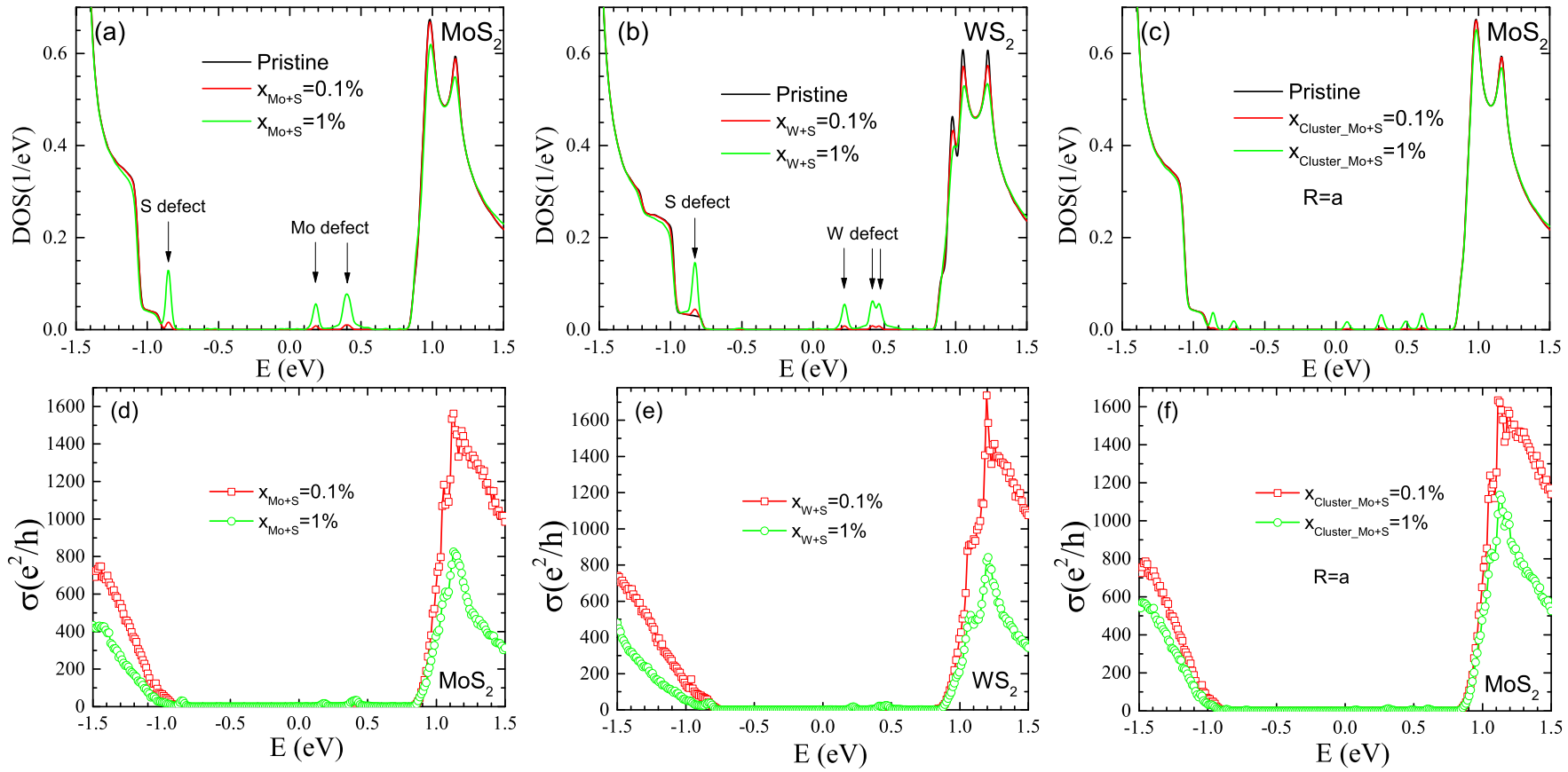

FIG. 2. (Color online) Top panels: DOS of (a) $\mathrm{MoS}_{2}$ and (b) $\mathrm{WS}_{2}$ with single defects, and of (c) $\mathrm{MoS}_{2}$ with clusters of defects with radius $R=a$. Panels (d)-(f) show the dc conductivity as a function of doping energy for the same concentrations of defects as in (a)-(c).

that the observations are done in multilayered samples). The fact that Mo and $\mathrm{W}$ defects lead to localized states well inside the gap, as illustrated by the densities of states also shown in Fig. 2, combined with the fact that the bands at the $K$ and $K^{\prime}$ points of the BZ can be approximated by an effective gapped Dirac equation [30,31], suggests that these defects can act as resonant scatterers [32], which give rise to a mobility almost independent of the carrier density
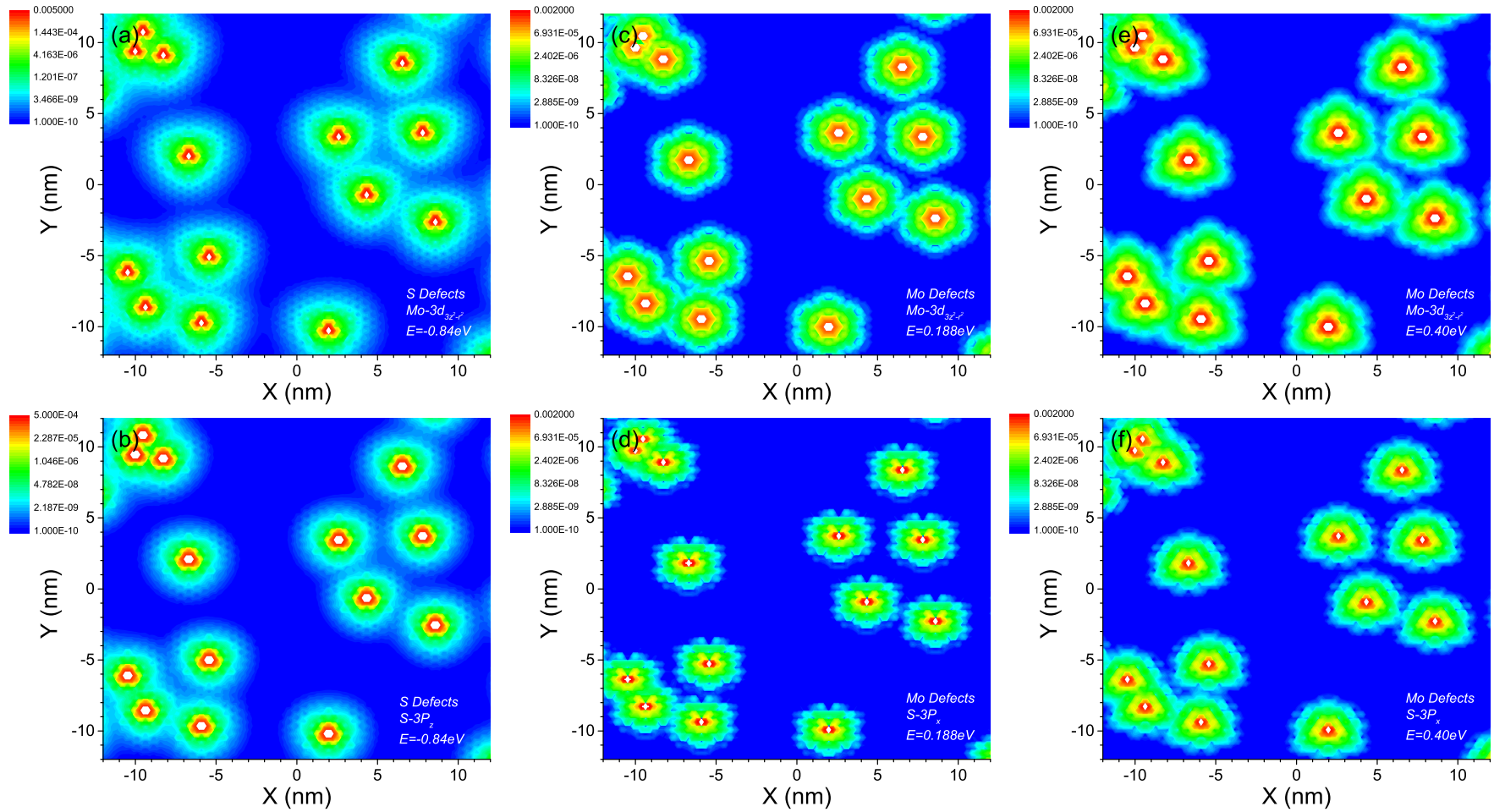

FIG. 3. (Color online) LDOS of for the corresponding dominant orbitals in the impurity states of $\mathrm{MoS}_{2}$ with single point defects. The white points indicate the position of the defects. The concentration of the defects is $0.1 \%$ for all panels. Results are obtained from the average of quasieigenstates for 100 samples with fixed distribution of point defects but different initial states. Please notice the different color scale in each panel. 


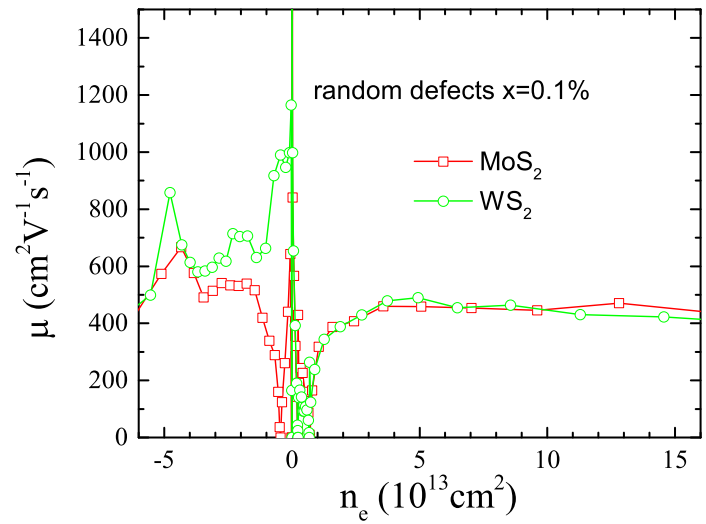

FIG. 4. (Color online) Mobility for $\mathrm{MoS}_{2}$ and $\mathrm{WS}_{2}$ for the same concentration $(0.1 \%)$ of point defects. $n$ doping corresponds to $\mathrm{Mo}(\mathrm{W})$ point defects and $p$ doping corresponds to $\mathrm{S}$ point defects.

(see below and the $T$-matrix analysis in the Supplemental Material [26]).

The localization of these midgap states is clearly seen by their local density of states (LDOS) plotted in Fig. 3. The amplitude of each orbital in real space is obtained from the average of quasiegenstates with different initial states. The profiles of the localized states show either hexagonal symmetry or mirror symmetry, depending on the type of the orbital and the energy of the midgap states. For $\mathrm{S}$ defects, the impurity state at the energy $-0.86 \mathrm{eV}$ is localized mainly ( $\sim 65.6 \%$ ) on $d_{3 z^{2}-r^{2}}$ orbitals of neighboring Mo atoms, with a small amount $(\sim 9.6 \%)$ on $p_{z, S}$ orbitals of neighboring $\mathrm{S}$ atoms. For Mo defects, on the other hand, there are two midgap states: one centered at $0.18 \mathrm{eV}$, with mainly localized $p_{x(y), A}(\sim 23.4 \%)$ and $d_{3 z^{2}-r^{2}}(\sim 21.2 \%)$ orbitals, and another centered at $0.4 \mathrm{eV}$, with mainly localized $d_{3 z^{2}-r^{2}}(\sim 46.4 \%)$ and $p_{x(y), A}(\sim 21.6 \%)$ orbitals. The detailed DOS of each orbital as a function of energy is shown in the Supplemental Material [26].

Results for the carrier mobility, defined as $\mu(E)=$ $\sigma(E) / e n_{e}(E)$, where the charge density $n_{e}(E)$ is obtained from the integral of density of states via $n_{e}(E)=\int_{0}^{E} \rho(\varepsilon) d \varepsilon$ are shown in Fig. 4. We notice that $n$ doping corresponds to $\mathrm{Mo}(\mathrm{W})$ point defects, whereas $p$ doping corresponds to $\mathrm{S}$ point defects. We observe that for $n$-doped samples, $\mathrm{MoS}_{2}$ and $\mathrm{WS}_{2}$ show similar mobilities, whereas for $p$-doped samples, the mobility of $\mathrm{WS}_{2}$ is larger than for $\mathrm{MoS}_{2}$. Our results show that in general, the mobilities of TMDs are low, but they are larger for holes than for electrons, in agreement with previous experimental results $[2,7]$. The results for the mobility suggest that it is independent of carrier concentration, except at the edge of the valence band. As we have discussed before, this is consistent with the expected features of resonant scatterers. A more detailed analysis of resonant scatterers in gapped Dirac systems is required in order to make this statement more quantitative, however. Note, finally, that our analysis leaves out the effect of the missing charge at the defect, which can lead to a long-range potential, and to intravalley scattering [33].

Conclusions. We have studied the effect of point defects in the DOS, optical and dc conductivity of single layers of TMDs such as $\mathrm{MoS}_{2}$ and $\mathrm{WS}_{2}$. The existence of point defects in the sample creates flat midgap bands which activate new optical transitions in the optical spectrum, leading to a background contribution which appears in the optical conductivity at low energies, in agreement with photoconductivity measurements. Our results show a significant asymmetry between electrons and holes. The dc conductivities and mobilities are larger for holes, in agreement with experiments, and we find higher mobilities for $p$-doped $\mathrm{WS}_{2}$ than for $\mathrm{MoS}_{2}$. Mo and $\mathrm{W}$ defects induce localized states well inside the gap, suggesting a behavior similar to that of resonant scatterers in graphene.

Acknowledgments. We thank the European Union Seventh Framework Programme under Grant Agreement No. 604391 Graphene Flagship. The support by the Stichting Fundamenteel Onderzoek der Materie (FOM) and the Netherlands National Computing Facilities Foundation (NCF) are acknowledged. S.Y. and M.I.K. acknowledge financial support from the European Research Council Advanced Grant program (Contract No. 338957). R.R. acknowledges financial support from the Juan de la Cierva Program (MEC, Spain). R.R. and F.G. acknowledge financial support from MINECO, Spain, through Grant No. FIS2011-23713, and the European Research Council Advanced Grant program (Contract No. 290846).
[1] S. Wu, J. S. Ross, G.-B. Liu, G. Aivazian, A. Jones, Z. Fei, W. Zhu, D. Xiao, W. Yao, D. Cobden et al., Nat. Phys. 9, 149 (2013).

[2] W. Bao, X. Cai, D. Kim, K. Sridhara, and M. S. Fuhrer, Appl. Phys. Lett. 102, 042104 (2013).

[3] S. Tongay, J. Suh, C. Ataca, W. Fan, A. Luce, J. S. Kang, J. Liu, C. Ko, R. Raghunathanan, J. Zhou et al., Sci. Rep. 3, 2657 (2013).

[4] W. Zhou, X. Zou, S. Najmaei, Z. Liu, Y. Shi, J. Kong, J. Lou, P. M. Ajayan, B. I. Yakobson, and J.-C. Idrobo, Nano Lett. 13, 2615 (2013).

[5] H. Qiu, T. Xu, Z. Wang, W. Ren, H. Nan, Z. Ni, Q. Chen, S. Yuan, F. Miao, F. Song et al., Nat. Commun. 4, 2642 (2013).
[6] A. N. Enyashin, M. Bar-Sadan, L. Houben, and G. Seifert, J. Phys. Chem. C 117, 10842 (2013).

[7] W. Zhu, T. Low, Y.-H. Lee, H. Wang, D. B. Farmer, J. Kong, F. Xia, and P. Avouris, Nat. Commun. 5, 3087 (2014).

[8] H. Schmidt, S. Wang, L. Chu, M. Toh, R. Kumar, W. Zhao, A. H. Castro Neto, J. Martin, S. Adam, B. Oezyilmaz et al., arXiv:1401.1063.

[9] C. Ataca and S. Ciraci, J. Phys. Chem. C 115, 13303 (2011).

[10] Y. Ma, Y. Dai, M. Guo, C. Niu, J. Lu, and B. Huang, Phys. Chem. Chem. Phys. 13, 15546 (2011).

[11] J.-w. Wei, Z.-w. Ma, H. Zeng, Z.-y. Wang, Q. Wei, and P. Peng, AIP Adv. 2, 042141 (2012) 
[12] H.-P. Komsa, J. Kotakoski, S. Kurasch, O. Lehtinen, U. Kaiser, and A. V. Krasheninnikov, Phys. Rev. Lett. 109, 035503 (2012).

[13] M. Ghorbani-Asl, A. N. Enyashin, A. Kuc, G. Seifert, and T. Heine, Phys. Rev. B 88, 245440 (2013).

[14] Y. Zhou, P. Yang, H. Zu, F. Gao, and X. Zu, Phys. Chem. Chem. Phys. 15, 10385 (2013).

[15] D. Liu, Y. Guo, L. Fang, and J. Robertson, Appl. Phys. Lett. 103, 183113 (2013).

[16] E. Cappelluti, R. Roldán, J. A. Silva-Guillén, P. Ordejón, and F. Guinea, Phys. Rev. B 88, 075409 (2013).

[17] R. Roldán, M. P. López-Sancho, E. Cappelluti, J. A. SilvaGuillén, P. Ordejón, and F. Guinea, arXiv:1401.5009.

[18] S. Yuan, H. De Raedt, and M. I. Katsnelson, Phys. Rev. B 82, 115448 (2010).

[19] T. O. Wehling, S. Yuan, A. I. Lichtenstein, A. K. Geim, and M. I. Katsnelson, Phys. Rev. Lett. 105, 056802 (2010).

[20] S. Yuan, R. Roldán, H. De Raedt, and M. I. Katsnelson, Phys. Rev. B 84, 195418 (2011).

[21] S. Yuan, T. O. Wehling, A. I. Lichtenstein, and M. I. Katsnelson, Phys. Rev. Lett. 109, 156601 (2012).

[22] K. F. Mak, C. Lee, J. Hone, J. Shan, and T. F. Heinz, Phys. Rev. Lett. 105, 136805 (2010).
[23] K. S. Novoselov, D. Jiang, F. Schedin, T. J. Booth, V. V. Khotkevich, S. V. Morozov, and A. K. Geim, Proc. Natl. Acad. Sci. USA 102, 10451 (2005).

[24] A. Splendiani, L. Sun, Y. Zhang, T. Li, J. Kim, C. Y. Chim, and F. Wang, Nano Lett. 10, 1271 (2010).

[25] A. Castellanos-Gomez, R. Roldán, E. Cappelluti, M. Buscema, F. Guinea, H. S. J. van der Zant, and G. A. Steele, Nano Lett. 13, 5361 (2013).

[26] See Supplemental Material at http://link.aps.org/supplemental/ 10.1103/PhysRevB.90.041402 for a detailed description of the arrangement of the defects in our calculation.

[27] Z. Li and J. P. Carbotte, Phys. Rev. B 86, 205425 (2012).

[28] H. Rostami and R. Asgari, Phys. Rev. B 89, 115413 (2014).

[29] Z. Li, E. A. Henriksen, Z. Jiang, Z. Hao, M. C. Martin, P. Kim, H. Stormer, and D. N. Basov, Nat. Phys. 4, 532 (2008).

[30] D. Xiao, G.-B. Liu, W. Feng, X. Xu, and W. Yao, Phys. Rev. Lett. 108, 196802 (2012).

[31] H.-Z. Lu, W. Yao, D. Xiao, and S.-Q. Shen, Phys. Rev. Lett. 110, 016806 (2013).

[32] T. Stauber, N. M. R. Peres, and F. Guinea, Phys. Rev. B 76, 205423 (2007).

[33] N. M. R. Peres, Rev. Mod. Phys. 82, 2673 (2010). 